\title{
Hilbert-type and Hardy-type integral inequalities with operator expressions and the best constants in the whole plane
}

\section{Xianyong Huang ${ }^{*}$, Junfei Cao, Bing He and Bicheng Yang}

\section{"Correspondence:}

huangxianyong@gdei.edu.cn Department of Mathematics, Guangdong University of Education, Xingangzhong Road, Guangzhou, 510303, P.R. China

\begin{abstract}
By introducing a mixed kernel function and the proper parameters, a new Hilbert-type integral inequality with a best constant factor in the whole plane is derived. As an application, the operator expressions, reverse inequalities, and Hardy-type inequalities are considered.
\end{abstract}

Keywords: parameter; Hilbert-type inequality; Hardy-type inequality; kernel; operator expression

\section{Introduction}

Suppose that $a_{m}, b_{n} \geq 0, a=\left\{a_{m}\right\}_{m=1}^{\infty}, b=\left\{b_{n}\right\}_{n=1}^{\infty} \in l^{2}\left(l^{2}:=\left\{x=\left\{x_{k}\right\}_{k=1}^{\infty}: \sum_{k=1}^{\infty} x_{k}^{2}<\infty\right\}\right)$, $\|a\|=\left\{\sum_{m=1}^{\infty} a_{m}^{2}\right\}^{\frac{1}{2}}>0,\|b\|>0$, we have the following well-known discrete Hilbert inequality [1]:

$$
\sum_{m=1}^{\infty} \sum_{n=1}^{\infty} \frac{a_{m} b_{n}}{m+n}<\pi\|a\|\|b\|
$$

where the constant factor $\pi$ is the best possible. Moreover, for $f(x), g(y) \geq 0, f, g \in L^{2}\left(\mathbf{R}_{+}\right)$, $\|f\|=\left(\int_{0}^{\infty} f^{2}(x) d x\right)^{\frac{1}{2}}>0,\|g\|>0$, we still have the following Hilbert integral inequality:

$$
\int_{0}^{\infty} \int_{0}^{\infty} \frac{f(x) g(y)}{x+y} d x d y<\pi\|f\|\|g\|
$$

with the same best constant factor $\pi$. Inequalities (1) and (2) are important in analysis and applications (cf. [2-12]).

All of the above integral inequalities are built in the quarter plane of the first quadrant. For some special kernel functions, it is meaningful to establish inequalities in the whole plane. In 2008, Yang [13] gave an inequality as follows:

$$
\begin{aligned}
& \int_{-\infty}^{\infty} \int_{-\infty}^{\infty} \frac{f(x) g(y)}{|1+x y|^{\lambda}} d x d y \\
& \quad<k_{\lambda}\left\{\int_{-\infty}^{\infty}|x|^{p\left(1-\frac{\lambda}{2}\right)-1} f^{p}(x) d x\right\}^{1 / p}\left\{\int_{-\infty}^{\infty}|y|^{q\left(1-\frac{\lambda}{2}\right)-1} g^{q}(y) d y\right\}^{1 / q},
\end{aligned}
$$

(c) 2015 Huang et al.; licensee Springer. This is an Open Access article distributed under the terms of the Creative Commons Attribution License (http://creativecommons.org/licenses/by/4.0), which permits unrestricted use, distribution, and reproduction in any medium, provided the original work is properly credited. 
where the constant factor $k_{\lambda}:=B\left(\frac{\lambda}{2}, \frac{\lambda}{2}\right)+2 B\left(1-\lambda, \frac{\lambda}{2}\right)$ is the best possible and $0<\lambda<1, p>1$, $(1 / p)+(1 / q)=1$. In 2010, Zeng and Xie [14] got the following inequality: If $p>1,(1 / p)+$ $(1 / q)=1, r \in(-1,0), 0<\alpha<\beta<\pi, f(x)$ and $g(y)$ are nonnegative measurable functions, satisfying $0<\int_{-\infty}^{\infty}|x|^{p\left(1-\frac{\lambda}{2}\right)-1} f^{p}(x) d x<\infty, 0<\int_{-\infty}^{\infty}|y|^{q\left(1-\frac{\lambda}{2}\right)-1} g^{q}(y) d y<\infty$, then

$$
\begin{aligned}
& \int_{-\infty}^{\infty} \int_{-\infty}^{\infty} f(x) g(y)\left|\ln \frac{x^{2}+2 x y \cos \alpha+y^{2}}{x^{2}+2 x y \cos \beta+y^{2}}\right| d x d y \\
& \quad<k\left\{\int_{-\infty}^{\infty}|x|^{p(1+r)-1} f^{p}(x) d x\right\}^{1 / p}\left\{\int_{-\infty}^{\infty}|y|^{q(1-r)-1} g^{q}(y) d y\right\}^{1 / q},
\end{aligned}
$$

where the constant factor

$$
k=\frac{4 \pi \sin [r(\beta-\alpha) / 2] \cos [(r / 2)(\pi-\alpha-\beta)]}{r \cos (r \pi / 2)}
$$

is the best possible. Subsequently, many scholars began to study the inequalities of this type (cf. [15-19]).

In 1934, Hardy [1] published the following Hardy integral inequality: If $p>1, f(x) \geq 0$, $f \in L^{p}\left(\mathbf{R}_{+}\right),\|f\|_{p}=\left\{\int_{0}^{\infty} f^{p}(x) d x\right\}^{1 / p}>0, F(x)=\int_{0}^{x} f(t) d t$, then

$$
\int_{0}^{\infty}\left(\frac{F(x)}{x}\right)^{p} d x<\left(\frac{p}{p-1}\right)^{p}\|f\|_{p}^{p}
$$

where the constant factor $[p /(p-1)]^{p}$ is the best possible. From Theorem 328, [1], it follows that

$$
\int_{0}^{\infty} F^{p}(x) d x<p^{p} \int_{0}^{\infty}(x f(x))^{p} d x
$$

where the constant factor $p^{p}$ is still the best possible and $F(x)=\int_{x}^{\infty} f(t) d t$. In 2009, Yang [20] gave the following best extensions of (6) by introducing an independent parameter $\lambda$. If $0<p<1, r>0, f \geq 0,0<\int_{0}^{\infty} x^{p\left(1+\frac{1-\lambda}{r}\right)-1} f^{p}(x) d x<\infty(\lambda \neq 1), F(x)=\int_{0}^{x} f(t) d t(\lambda>1)$, $F(x)=\int_{x}^{\infty} f(t) d t(\lambda<1)$, then

$$
\int_{0}^{\infty} x^{\frac{p}{r}(1-\lambda)-1} F^{p}(x) d x>\left(\frac{r}{|1-\lambda|}\right)^{p} \int_{0}^{\infty} x^{\frac{p}{r}(1-\lambda)-1}(x f(x))^{p} d x
$$

where the constant factor $\left(\frac{r}{|1-\lambda|}\right)^{p}$ is the best possible.

The main objective of this paper is to establish inequalities in the whole plane, as well as the corresponding operator expression, the equivalent form, and the reverse inequality. Special emphasis is placed on a Hardy-type inequality with the best possible constant.

\section{Some lemmas}

In what follows, $\alpha_{1}, \alpha_{2}$ will be real numbers such that $0<\alpha_{1} \leq \alpha_{2}<\pi, \delta \in\{-1,1\}, \lambda_{1}, \lambda_{2}<0$, $\lambda_{1}+\lambda_{2}=-\lambda$, and

$$
\begin{aligned}
& h\left(x^{\delta} y\right):=\min _{i \in\{1,2\}} \min \left\{1,\left[\left|x^{\delta} y\right|^{\gamma}+\left(x^{\delta} y\right)^{\gamma} \cos \alpha_{i}\right]^{\lambda / \gamma}\right\}, \\
& k_{\lambda}(x, y):=\min _{i \in\{1,2\}} \min \left\{|x|,\left[|y|^{\gamma}+y^{\gamma} \operatorname{sgn} x \cos \alpha_{i}\right]^{\lambda / \gamma}\right\} .
\end{aligned}
$$


Lemma 1 If $C(\lambda):=\frac{\lambda}{\lambda_{1} \lambda_{2}} \cdot 2^{\lambda_{2} / \gamma}\left[\left(\cos \frac{\alpha_{1}}{2}\right)^{2 \lambda_{2} / \gamma}+\left(\sin \frac{\alpha_{2}}{2}\right)^{2 \lambda_{2} / \gamma}\right]$, the weight function

$$
\begin{aligned}
& \omega_{\delta}\left(\lambda_{2}, y\right):=\int_{-\infty}^{\infty} h\left(x^{\delta} y\right) \frac{|y|^{\lambda_{2}}}{|x|^{1-\delta \lambda_{2}}} d x, \\
& \varpi_{\delta}\left(\lambda_{2}, x\right):=\int_{-\infty}^{\infty} h\left(x^{\delta} y\right) \frac{|x|^{\delta \lambda_{2}}}{|y|^{1-\lambda_{2}}} d y,
\end{aligned}
$$

then for all $x, y \neq 0$, we have

$$
\omega_{\delta}\left(\lambda_{2}, y\right)=\varpi_{\delta}\left(\lambda_{2}, x\right)=C(\lambda) .
$$

Proof (i) For $\delta=1$, setting $u=x y$, we find for $y \neq 0$,

$$
\begin{aligned}
\omega_{1}\left(\lambda_{2}, y\right)= & \int_{-\infty}^{\infty} h(u)|u|^{\lambda_{2}-1} d u \\
= & \int_{0}^{\infty} \min _{i \in\{1,2\}} \min \left\{1, u^{\lambda}\left(1+\cos \alpha_{i}\right)^{\lambda / \gamma}\right\} u^{\lambda_{2}-1} d u \\
& +\int_{0}^{\infty} \min _{i \in\{1,2\}} \min \left\{1, u^{\lambda}\left(1-\cos \alpha_{i}\right)^{\lambda / \gamma}\right\} u^{\lambda_{2}-1} d u \\
= & \frac{1}{\lambda}\left[\int_{0}^{\infty} \min _{i \in\{1,2\}}\left(1+\cos \alpha_{i}\right)^{\lambda_{2} / \gamma} v^{\lambda_{2} / \lambda-1} \min \{1, v\} d v\right. \\
& \left.+\int_{0}^{\infty} \min _{i \in\{1,2\}}\left(1-\cos \alpha_{i}\right)^{\lambda_{2} / \gamma} v^{\lambda_{2} / \lambda-1} \min \{1, v\} d v\right] \\
= & \frac{1}{\lambda}\left[\left(1+\cos \alpha_{1}\right)^{\lambda_{2} / \gamma}+\left(1-\cos \alpha_{2}\right)^{\lambda_{2} / \gamma}\right] \int_{0}^{\infty} v^{\lambda_{2} / \lambda-1} \min \{1, v\} d v \\
= & \frac{\lambda}{\lambda_{1} \lambda_{2}} \cdot 2^{\lambda_{2} / \gamma}\left[\left(\cos \frac{\alpha_{1}}{2}\right)^{2 \lambda_{2} / \gamma}+\left(\sin \frac{\alpha_{2}}{2}\right)^{2 \lambda_{2} / \gamma}\right]=C(\lambda) .
\end{aligned}
$$

(ii) For $\delta=-1$, setting $u=y / x$, we still have $\omega_{-1}\left(\lambda_{2}, y\right)=C(\lambda)$.

Setting $u=x^{\delta} y$, we get

$$
\varpi_{\delta}\left(\lambda_{2}, x\right)=\int_{-\infty}^{\infty} h(u)|u|^{\lambda_{2}-1} d u=C(\lambda) .
$$

Hence we have (10).

Remark 1 Let $\alpha_{1}=\alpha_{2}=\alpha$, then $h(u)=\min \left\{1,\left[|u|^{\gamma}+(u)^{\gamma} \cos \alpha\right]^{\lambda / \gamma}\right\}$ and

$$
C(\lambda)=\frac{\lambda}{\lambda_{1} \lambda_{2}} \cdot 2^{\lambda_{2} / \gamma}\left[\left(\cos \frac{\alpha}{2}\right)^{2 \lambda_{2} / \gamma}+\left(\sin \frac{\alpha}{2}\right)^{2 \lambda_{2} / \gamma}\right] .
$$

Lemma 2 If $p>1, \frac{1}{p}+\frac{1}{q}=1, f(x)$ is a nonnegative measurable function in $\mathbf{R}$, then (i) for $p>1$, we have

$$
\begin{aligned}
J & :=\int_{-\infty}^{\infty}|y|^{p \lambda_{2}-1}\left[\int_{-\infty}^{\infty} h\left(x^{\delta} y\right) f(x) d x\right]^{p} d y \\
& \leq C^{p}(\lambda) \int_{-\infty}^{\infty}|x|^{p\left(1-\delta \lambda_{2}\right)-1} f^{p}(x) d x
\end{aligned}
$$

(ii) for $0<p<1$, we have the reverse of (11). 
Proof For $p>1$, by the Hölder inequality with weight [21], we have

$$
\begin{aligned}
& {\left[\int_{-\infty}^{\infty} h\left(x^{\delta} y\right) f(x) d x\right]^{p}} \\
& \quad=\left[\int_{-\infty}^{\infty} h\left(x^{\delta} y\right)\left(\frac{|x|^{\left(1-\delta \lambda_{2}\right) / q}}{|y|^{\left(1-\lambda_{2}\right) / p}} f(x)\right)\left(\frac{|y|^{\left(1-\lambda_{2}\right) / p}}{|x|^{\left(1-\delta \lambda_{2}\right) / q}}\right) d x\right]^{p} \\
& \quad \leq \int_{-\infty}^{\infty} h\left(x^{\delta} y\right) \frac{|x|^{\left(1-\delta \lambda_{2}\right)(p-1)}}{|y|^{1-\lambda_{2}}} f^{p}(x) d x\left[\int_{-\infty}^{\infty} h\left(x^{\delta} y\right) \frac{|y|^{\left(1-\lambda_{2}\right)(q-1)}}{|x|^{1-\delta \lambda_{2}}} d x\right]^{p-1} \\
& \quad=\frac{\left[\omega_{\delta}\left(\lambda_{2}, y\right)\right]^{p-1}}{|y|^{p \lambda_{2}-1}} \int_{-\infty}^{\infty} h\left(x^{\delta} y\right) \frac{|x|^{\left(1-\delta \lambda_{2}\right)(p-1)}}{|y|^{1-\lambda_{2}}} f^{p}(x) d x .
\end{aligned}
$$

Then by (10) and the Fubini theorem [22], it follows that

$$
\begin{aligned}
J & \leq C^{p-1}(\lambda) \int_{-\infty}^{\infty}\left[\int_{-\infty}^{\infty} h\left(x^{\delta} y\right) \frac{|x|^{\left(1-\delta \lambda_{2}\right)(p-1)}}{|y|^{1-\lambda_{2}}} f^{p}(x) d x\right] d y \\
& =C^{p-1}(\lambda) \int_{-\infty}^{\infty} \omega_{\delta}\left(\lambda_{2}, x\right)|x|^{p\left(1-\delta \lambda_{2}\right)-1} f^{p}(x) d x \\
& =C^{p}(\lambda) \int_{-\infty}^{\infty}|x|^{p\left(1-\delta \lambda_{2}\right)-1} f^{p}(x) d x
\end{aligned}
$$

and so (11) is proved.

(ii) For $0<p<1$, by the reverse Hölder inequality [21], in view of the assumptions and with the same way, we obtain the reverse of (11).

\section{Main results and applications}

In this section, we set the functions and spaces as follows:

$$
\begin{aligned}
& \varphi(x):=|x|^{p\left(1-\delta \lambda_{2}\right)-1} \quad(x \in \mathbf{R}), \quad \psi(y):=|y|^{q\left(1-\lambda_{2}\right)-1} \quad(y \in \mathbf{R}), \\
& L_{p, \varphi}(\mathbf{R}):=\left\{f:\|f\|_{p, \varphi}=\left\{\int_{-\infty}^{\infty} \varphi(x)|f(x)|^{p} d x\right\}^{1 / p}<\infty\right\}, \\
& L_{q, \psi}(\mathbf{R}):=\left\{g:\|g\|_{q, \psi}=\left\{\int_{-\infty}^{\infty} \psi(y)|g(y)|^{q} d y\right\}^{1 / q}<\infty\right\} .
\end{aligned}
$$

Theorem 1 If $p>1, \frac{1}{p}+\frac{1}{q}=1, f(x), g(y) \geq 0$, satisfying $f(x) \in L_{p, \varphi}(\mathbf{R}), g(y) \in L_{q, \psi}(\mathbf{R})$, $\|f\|_{p, \varphi},\|g\|_{q, \psi}>0$, then we have the following equivalent inequalities:

$$
\begin{aligned}
& I:=\int_{-\infty}^{\infty} \int_{-\infty}^{\infty} h\left(x^{\delta} y\right) f(x) g(y) d x d y<C(\lambda)\|f\|_{p, \varphi}\|g\|_{q, \psi}, \\
& J=\int_{-\infty}^{\infty}|y|^{\lambda_{2}-1}\left[\int_{-\infty}^{\infty} h\left(x^{\delta} y\right) f(x) d x\right]^{p} d y<C^{p}(\lambda)\|f\|_{p, \varphi}^{p},
\end{aligned}
$$

where the constant factors $C(\lambda)$ and $C^{p}(\lambda)$ in the above inequalities are the best possible.

Proof If (12) takes the form of equality for a $y \neq 0$, then there exist constants $A$ and $B$, such that they are not all zero, and

$$
A \frac{|x|^{\left(1-\delta \lambda_{2}\right)(p-1)}}{|y|^{1-\lambda_{2}}} f^{p}(x)=B \frac{|y|^{\left(1-\lambda_{2}\right)(q-1)}}{|x|^{1-\delta \lambda_{2}}} \quad \text { a.e. in } \mathbf{R} \text {. }
$$


We confirm that $A \neq 0$ (otherwise $B=A=0$ ), from which

$$
|x|^{p\left(1-\delta \lambda_{2}\right)-1} f^{p}(x)=|y|^{q\left(1-\lambda_{2}\right)} \frac{B}{A|x|} \quad \text { a.e. in } \mathbf{R},
$$

in view of $\int_{-\infty}^{\infty} \frac{1}{|x|} d x=\infty$, which contradicts the fact that $\|f\|_{p, \varphi}>0$. Hence (12) takes a strict inequality, so does (11), thus (14) is valid.

By the Hölder inequality with weight [21], we obtain

$$
I=\int_{-\infty}^{\infty}\left[|y|^{\lambda_{2}-\frac{1}{p}} \int_{-\infty}^{\infty} h\left(x^{\delta} y\right) f(x) d x\right]\left(|y|^{\frac{1}{p}-\lambda_{2}} g(y) d y\right) \leq J^{1 / p}\|g\|_{q, \psi} .
$$

By (14), we have (13). On the other hand, suppose that (13) is valid, setting

$$
g(y):=|y|^{p \lambda_{2}-1}\left[\int_{-\infty}^{\infty} h\left(x^{\delta} y\right) f(x) d x\right]^{p-1} \quad(y \in \mathbf{R} \backslash\{0\}),
$$

then we find $J=\|g\|_{q, \psi}^{q}$. By (11), we have $J<\infty$. If $J=0$, then (14) is valid trivially; if $0<J<\infty$, then by (13), it follows that

$$
\begin{aligned}
& 0<\|g\|_{q, \psi}^{q}=J=I<C(\lambda)\|f\|_{p, \varphi}\|g\|_{q, \psi}<\infty, \\
& J^{1 / p}=\|g\|_{q, \psi}^{q / p}<C(\lambda)\|f\|_{p, \varphi},
\end{aligned}
$$

and then (14) follows, which is equivalent to (13).

For any $n>\frac{1}{p\left|\lambda_{2}\right|}(n \in \mathbf{N})$, setting $E_{\delta}:=\left\{x \in \mathbf{R}:|x|^{\delta} \geq 1\right\}$ and

$$
\tilde{f}(x):=\left\{\begin{array}{ll}
|x|^{\delta\left(\lambda_{2}-\frac{1}{n p}\right)-1}, & x \in E_{\delta}, \\
0, & x \in \mathbf{R} \backslash E_{\delta},
\end{array} \quad \tilde{g}(y):= \begin{cases}|y|^{\lambda_{2}+\frac{1}{n q}-1}, & y \in[-1,1], \\
0, & y \in(-\infty,-1) \cup(1, \infty),\end{cases}\right.
$$

then

$$
\begin{aligned}
\widetilde{L} & :=\|\widetilde{f}\|_{p, \varphi}\|\widetilde{g}\|_{q, \psi}=\left\{\int_{E_{\delta}}|x|^{-\frac{\delta}{n}-1} d x\right\}^{1 / p}\left\{\int_{-1}^{1}|y|^{\frac{1}{n}-1} d y\right\}^{1 / q} \\
& =2\left\{\int_{\left\{x>0: x^{\delta} \geq 1\right\}} x^{-\frac{\delta}{n}-1} d x\right\}^{1 / p}\left\{\int_{0}^{1} y^{\frac{1}{n}-1} d y\right\}^{1 / q}=2 n .
\end{aligned}
$$

Suppose that $I(x):=\int_{-1}^{1} h\left(x^{\delta} y\right)|y|^{\lambda_{2}+\frac{1}{n q}-1} d y$, we confirm that

$$
I(x)=|x|^{-\delta \lambda_{2}-\frac{\delta}{n q}} \int_{0}^{|x|^{\delta}}(h(v)+h(-v)) v^{\lambda_{2}+\frac{1}{n q}-1} d v .
$$

In fact, for $x>0$,

$$
\begin{aligned}
I(x) & =\int_{-1}^{0} h\left(x^{\delta} y\right)(-y)^{\lambda_{2}+\frac{1}{n q}-1} d y+\int_{0}^{1} h\left(x^{\delta} y\right) y^{\lambda_{2}+\frac{1}{n q}-1} d y \\
& =x^{-\delta \lambda_{2}-\frac{\delta}{n q}} \int_{0}^{x^{\delta}} h(-v) v^{\lambda_{2}+\frac{1}{n q}-1} d v+x^{-\delta \lambda_{2}-\frac{\delta}{n q}} \int_{0}^{x^{\delta}} h(v) v^{\lambda_{2}+\frac{1}{n q}-1} d v \\
& =x^{-\delta \lambda_{2}-\frac{\delta}{n q}} \int_{0}^{x^{\delta}}(h(v)+h(-v)) v^{\lambda_{2}+\frac{1}{n q}-1} d v .
\end{aligned}
$$


Similarly, we get

$$
\begin{aligned}
I(x) & =\int_{-1}^{0} h\left(x^{\delta} y\right)(-y)^{\lambda_{2}+\frac{1}{n q}-1} d y+\int_{0}^{1} h\left(x^{\delta} y\right) y^{\lambda_{2}+\frac{1}{n q}-1} d y \\
& =(-x)^{-\delta \lambda_{2}-\frac{\delta}{n q}} \int_{0}^{(-x)^{\delta}}(h(v)+h(-v)) v^{\lambda_{2}+\frac{1}{n q}-1} d v
\end{aligned}
$$

for $x<0$.

Applying the Fubini theorem [22],

$$
\begin{aligned}
& \widetilde{I}:=\int_{-\infty}^{\infty} \int_{-\infty}^{\infty} h\left(x^{\delta} y\right) \tilde{f}(x) \widetilde{g}(y) d x d y \\
& =\int_{E_{\delta}}|x|^{\delta\left(\lambda_{2}-\frac{1}{n p}\right)-1}\left(\int_{-1}^{1} h\left(x^{\delta} y\right)|y|^{\lambda_{2}+\frac{1}{n q}-1} d y\right) d x \\
& =\int_{E_{\delta}}|x|^{-\frac{\delta}{n}-1}\left(\int_{0}^{|x|^{\delta}}(h(u)+h(-u)) u^{\lambda_{2}+\frac{1}{n q}-1} d u\right) d x \\
& =\int_{E_{\delta}}|x|^{-\frac{\delta}{n}-1}\left(\int_{0}^{1}(h(u)+h(-u)) u^{\lambda_{2}+\frac{1}{n q}-1} d u\right) d x \\
& +\int_{E_{\delta}}|x|^{-\frac{\delta}{n}-1}\left(\int_{1}^{|x|^{\delta}}(h(u)+h(-u)) u^{\lambda_{2}+\frac{1}{n q}-1} d u\right) d x \\
& =2 n \int_{0}^{1}(h(u)+h(-u)) u^{\lambda_{2}+\frac{1}{n q}-1} d u \\
& +\int_{1}^{\infty}\left(\int_{\left\{x:|x|^{\delta} \geq u\right\}}^{1}|x|^{-\frac{\delta}{n}-1} d x\right)(h(u)+h(-u)) u^{\lambda_{2}+\frac{1}{n q}-1} d u \\
& =2 n\left[\int_{0}^{1}(h(u)+h(-u)) u^{\lambda_{2}+\frac{1}{n q}-1} d u+\int_{1}^{\infty}(h(u)+h(-u)) u^{\lambda_{2}-\frac{1}{n p}-1} d u\right] \text {. }
\end{aligned}
$$

If there exists a constant $K \leq C(\lambda)$, such that (13) is still valid when replacing $C(\lambda)$ by $K$, and $f(x), g(y)$ by $\tilde{f}(x), \tilde{g}(y)$, respectively, then it gives

$$
\begin{aligned}
& \int_{0}^{1}(h(u)+h(-u)) u^{\lambda_{2}+\frac{1}{n q}-1} d u+\int_{1}^{\infty}(h(u)+h(-u)) u^{\lambda_{2}-\frac{1}{n p}-1} d u \\
& \quad=\frac{1}{2 n} \widetilde{I}<\frac{1}{2 n} k \widetilde{L}=K .
\end{aligned}
$$

Since $\left\{u^{\lambda_{2}+\frac{1}{n q}-1}\right\}(0<u \leq 1)$ and $\left\{u^{\lambda_{2}-\frac{1}{n p}-1}\right\}(u>1)$ are nonnegative, monotonically increasing, by the Levi theorem [22],

$$
\begin{aligned}
C(\lambda) & =\int_{0}^{1} \lim _{n \rightarrow \infty}(h(u)+h(-u)) u^{\lambda_{2}+\frac{1}{n q}-1} d u+\int_{1}^{\infty} \lim _{n \rightarrow \infty}(h(u)+h(-u)) u^{\lambda_{2}-\frac{1}{n p}-1} d u \\
& =\lim _{n \rightarrow \infty}\left[\int_{0}^{1}(h(u)+h(-u)) u^{\lambda_{2}+\frac{1}{n q}-1} d u+\int_{1}^{\infty}(h(u)+h(-u)) u^{\lambda_{2}-\frac{1}{n p}-1} d u\right] \leq K .
\end{aligned}
$$

Hence $C(\lambda)=K$ in (13) is the best possible. 
By the equivalence, the constant factor $C^{p}(\lambda)$ in (14) is still the best possible. Otherwise, we would reach a contradiction by (15): that the constant in (13) is not the best possible.

Remark 2 (i) With the assumptions of Theorem 1, we define a Hilbert-type integral operator $T: L_{p, \varphi}(\mathbf{R}) \rightarrow L_{p, \psi^{1-p}}(\mathbf{R})$ as follows: For any $f \in L_{p, \varphi}(\mathbf{R})$, there exists a $T$, satisfying, for any $y \neq 0$,

$$
(T f)(y)=\int_{-\infty}^{\infty} h\left(x^{\delta} y\right) f(x) d x
$$

By (14), we obtain

$$
\|T f\|_{p, \psi^{1-p}} \leq C(\lambda)\|f\|_{p, \varphi}
$$

from which $T f \in L_{p, \psi^{1-p}}(\mathbf{R})$. Hence $T$ is a bounded linear operator with $\|T\| \leq C(\lambda)$. Since the constant factor in (14) is the best possible, we have

$$
\|T\|:=\sup _{f(\neq \theta) \in L_{p, \varphi}(\mathbf{R})} \frac{\|T f\|_{p, \psi^{1-p}}}{\|f\|_{p, \varphi}}=C(\lambda)
$$

(ii) For $\delta=1$, (13) and (14) are reduced to the following inequalities with non-homogeneous kernel and the best possible constant factors:

$$
\begin{aligned}
& \int_{-\infty}^{\infty} \int_{-\infty}^{\infty} h(x y) f(x) g(y) d x d y \\
& <C(\lambda)\left\{\int_{-\infty}^{\infty}|x|^{p\left(1-\lambda_{2}\right)-1} f^{p}(x) d x\right\}^{1 / p}\left\{\int_{-\infty}^{\infty}|y|^{q\left(1-\lambda_{2}\right)-1} g^{q}(y) d y\right\}^{1 / q}, \\
& \int_{-\infty}^{\infty}|y|^{p \lambda_{2}-1}\left[\int_{-\infty}^{\infty} h(x y) f(x) d x\right]^{p} d y<C^{p}(\lambda) \int_{-\infty}^{\infty}|x|^{p\left(1-\lambda_{2}\right)-1} f^{p}(x) d x
\end{aligned}
$$

(iii) For $\delta=-1$ in (13) and (14), replacing $|x|^{\lambda} f(x)$ by $f(x)$ gives the following inequalities with homogeneous kernel and the best possible constant factors $\left(k_{\lambda}(x, y):=\right.$ $\left.\min _{i \in\{1,2\}} \min \left\{|x|,\left[|y|^{\gamma}+y^{\gamma} \operatorname{sgn} x \cos \alpha_{i}\right]^{\lambda / \gamma}\right\}\right):$

$$
\begin{aligned}
& \int_{-\infty}^{\infty} k_{\lambda}(x, y) f(x) g(y) d x d y \\
& <C(\lambda)\left\{\int_{-\infty}^{\infty}|x|^{p\left(1-\lambda_{1}\right)-1} f^{p}(x) d x\right\}^{1 / p}\left\{\int_{-\infty}^{\infty}|y|^{q\left(1-\lambda_{2}\right)-1} g^{q}(y) d y\right\}^{1 / q}, \\
& \int_{-\infty}^{\infty}|y|^{p \lambda_{2}-1}\left[\int_{-\infty}^{\infty} k_{\lambda}(x, y) f(x) d x\right]^{p} d y<C^{p}(\lambda) \int_{-\infty}^{\infty}|x|^{p\left(1-\lambda_{1}\right)-1} f^{p}(x) d x
\end{aligned}
$$

Theorem 2 With the assumptions of Theorem 1, replacing $p>1$ by $0<p<1$, we have the following equivalent reverse inequalities:

$$
I=\int_{-\infty}^{\infty} \int_{-\infty}^{\infty} h\left(x^{\delta} y\right) f(x) g(y) d x d y>C(\lambda)\|f\|_{p, \varphi}\|g\|_{q, \psi},
$$




$$
J=\int_{-\infty}^{\infty}|y|^{p \lambda_{2}-1}\left[\int_{-\infty}^{\infty} h\left(x^{\delta} y\right) f(x) d x\right]^{p} d y>C^{p}(\lambda)\|f\|_{p, \varphi}^{p}
$$

where the constant factors $C(\lambda)$ and $C^{p}(\lambda)$ in the above inequalities are the best possible.

Proof The proof is similar to the proof of Theorem 1 and hence by Lemma 2 with $0<p<1$, we have the reverses of (13), (14), and (15), thus (19) and (20) are valid. On the other hand, suppose that (19) is valid. Let $g(y)$ be the same as in Theorem 1, then $J>0$ with the reverse of (11). If $J=\infty$, then (20) is obviously valid; if $0<J<\infty$, then by (19), we obtain the reverses of (16) and (17). Hence we have (20), which is equivalent to (19).

If the constant factor $C(\lambda)$ in (19) is not the best possible, then there exists a positive constant $k$, with $k \geq C(\lambda)$, such that (19) is still valid when replacing $C(\lambda)$ by $k$. There exists $0<a<2 \lambda_{2}$, such that $n \geq \frac{2}{a|q|}$, we have the reverse of (18):

$$
\int_{0}^{1}(h(u)+h(-u)) u^{\lambda_{2}+\frac{1}{n q}-1} d u+\int_{1}^{\infty}(h(u)+h(-u)) u^{\lambda_{2}-\frac{1}{n p}-1} d u>k .
$$

Since

$$
0 \leq(h(u)+h(-u)) u^{\lambda_{2}-\frac{1}{n p}-1} \leq(h(u)+h(-u)) u^{\lambda_{2}-\frac{1}{(n+1) p}-1} \quad(u \in[1, \infty)),
$$

then by the Levi theorem,

$$
\begin{aligned}
& \int_{1}^{\infty}(h(u)+h(-u)) u^{\lambda_{2}-\frac{1}{n p}-1} d u \\
& \quad=\int_{1}^{\infty}(h(u)+h(-u)) u^{\lambda_{2}-1} d u+o(1) \quad(n \rightarrow \infty) .
\end{aligned}
$$

In view of $q<0$ and $n \geq \frac{2}{a|q|}$, we have

$$
\begin{aligned}
& (h(u)+h(-u)) u^{\lambda_{2}+\frac{1}{n q}-1} \leq(h(u)+h(-u)) u^{\lambda_{2}-\frac{a}{2}-1} \quad(u \in(0,1]), \\
& 0 \leq \int_{0}^{1}(h(u)+h(-u)) u^{\lambda_{2}-\frac{a}{2}-1} d u \leq \int_{0}^{\infty}(h(u)+h(-u)) u^{\lambda_{2}-\frac{a}{2}-1} d u \\
& =\frac{-4 \lambda}{\left(a+2 \lambda_{1}\right)\left(a-2 \lambda_{2}\right)} \cdot 2^{\lambda_{2} / \gamma}\left[\left(\cos \frac{\alpha_{1}}{2}\right)^{2 \lambda_{2} / \gamma}+\left(\sin \frac{\alpha_{2}}{2}\right)^{2 \lambda_{2} / \gamma}\right]<\infty .
\end{aligned}
$$

By the Lebesgue dominated convergence theorem [22], we have

$$
\int_{0}^{1}(h(u)+h(-u)) u^{\lambda_{2}+\frac{1}{n q}-1} d u=\int_{0}^{1}(h(u)+h(-u)) u^{\lambda_{2}-1} d u+o(1) \quad(n \rightarrow \infty) .
$$

Therefore, we get $C(\lambda) \geq K$. Hence $C(\lambda)=K$ is the best possible constant factor of (19).

By the equivalence, the constant factor $C^{p}(\lambda)$ in (20) is still the best possible. Otherwise, we would reach a contradiction by the reverse of (15): that the constant in (19) is not the best possible.

As an application of Hilbert-type inequality, suppose that $\delta=1$,

$$
h(x y)=\min _{i \in\{1,2\}} \min \left\{1,\left[|x y|^{\gamma}+(x y)^{\gamma} \cos \alpha_{i}\right]^{\lambda / \gamma}\right\},
$$


$h(u)=0(|u|>1)$, i.e. $h(x y)=0\left(|x|>\frac{1}{|y|}\right)$, then we have

$$
C(\lambda)=C_{1}(\lambda):=\int_{-1}^{1} h(u)|u|^{\lambda_{2}-1} d u=\int_{0}^{1}(h(u)+h(-u)) u^{\lambda_{2}-1} d u
$$

and we get Hardy-type inequality with the non-homogeneous kernel as follows.

Corollary 1 If $p>1, \frac{1}{p}+\frac{1}{q}=1, C_{1}(\lambda)=\int_{-1}^{1} h(u)|u|^{\lambda_{2}-1} d u, f(x), g(y) \geq 0$, satisfying $0<$ $\int_{-\infty}^{\infty}|x|^{p\left(1-\lambda_{2}\right)-1} f^{p}(x) d x<\infty, 0<\int_{-\infty}^{\infty}|y|^{q\left(1-\lambda_{2}\right)-1} g^{q}(y) d y<\infty$, then we have the following equivalent inequalities:

$$
\begin{gathered}
\int_{-\infty}^{\infty}\left(\int_{\frac{-1}{|y|}}^{\frac{1}{|y|}} h(x y) f(x) d x\right) g(y) d y=\int_{-\infty}^{\infty}\left(\int_{\frac{-1}{|x|}}^{\frac{1}{|x|}} h(x y) g(y) d y\right) f(x) d x \\
<C_{1}(\lambda)\left\{\int_{-\infty}^{\infty}|x|^{p\left(1-\lambda_{2}\right)-1} f^{p}(x) d x\right\}^{1 / p}\left\{\int_{-\infty}^{\infty}|y|^{q\left(1-\lambda_{2}\right)-1} g^{q}(y) d y\right\}^{1 / q} \\
\int_{-\infty}^{\infty}|y|^{p \lambda_{2}-1}\left(\int_{\frac{-1}{|y|}}^{\frac{1}{|y|}} h(x y) f(x) d x\right)^{p} d y<C_{1}^{p}(\lambda) \int_{-\infty}^{\infty}|x|^{p\left(1-\lambda_{2}\right)-1} f^{p}(x) d x
\end{gathered}
$$

where the constant factors $C_{1}(\lambda)$ and $C_{1}^{p}(\lambda)$ in the above inequalities are the best possible. If $0<p<1$, then we have the reverse equivalent inequalities with the same best possible constant factors.

Similarly, if $h(u)=0(|u|<1)$, then $h(x y)=0\left(|x|<\frac{1}{|y|}\right)$. Setting

$$
E_{x^{-1}}=\left\{y \in \mathbf{R}: y \leq-\frac{1}{|x|} \text { or } y \geq \frac{1}{|x|}\right\}
$$

and we get another Hardy-type inequality with the non-homogeneous kernel as follows:

Corollary 2 If $p>1, \frac{1}{p}+\frac{1}{q}=1$,

$$
C_{2}(\lambda)=\int_{1}^{\infty}(h(u)+h(-u)) u^{\lambda_{2}-1} d u
$$

$f(x), g(y) \geq 0$, such that $0<\int_{-\infty}^{\infty}|x|^{p\left(1-\lambda_{1}\right)-1} f^{p}(x) d x<\infty$,

$$
0<\int_{-\infty}^{\infty}|y|^{q\left(1-\lambda_{2}\right)-1} g^{q}(y) d y<\infty
$$

then we have the following equivalent inequalities with the best possible constant factors:

$$
\begin{aligned}
& \int_{-\infty}^{\infty}\left(\int_{E_{y^{-1}}} h(x y) f(x) d x\right) g(y) d y \\
& \quad=\int_{-\infty}^{\infty}\left(\int_{E_{x^{-1}}} h(x y) g(y) d y\right) f(x) d x \\
& \quad<C_{2}(\lambda)\left\{\int_{-\infty}^{\infty}|x|^{p\left(1-\lambda_{1}\right)-1} f^{p}(x) d x\right\}^{1 / p}\left\{\int_{-\infty}^{\infty}|y|^{q\left(1-\lambda_{2}\right)-1} g^{q}(y) d y\right\}^{1 / q},
\end{aligned}
$$




$$
\begin{gathered}
\int_{-\infty}^{\infty}|y|^{p \lambda_{2}-1}\left(\int_{E_{y^{-1}}} k_{\lambda}(x, y) f(x) d x\right)^{p} d y \\
<C_{2}^{p}(\lambda) \int_{-\infty}^{\infty}|x|^{p\left(1-\lambda_{1}\right)-1} f^{p}(x) d x .
\end{gathered}
$$

If $0<p<1$, then we get the reverse equivalent inequalities with the same best possible constant factors.

\section{Competing interests}

The authors declare that they have no competing interests.

\section{Authors' contributions}

XH carried out the mathematical studies, participated in the sequence alignment and drafted the manuscript. JC participated in the design of the study and performed the numerical analysis. All authors read and approved the final manuscript.

\section{Acknowledgements}

This work is supported by the National Natural Science Foundation of China (No. 11301090), Training Plan Fund of Outstanding Young Teachers of Higher Learning Institutions of Guangdong Province in China (No. Yq20145084602) and the Natural Science Foundation of Guangdong University of Education (No. 2014yjxm05).

\section{Received: 17 January 2015 Accepted: 1 April 2015 Published online: 11 April 2015}

\section{References}

1. Hardy, GH, Littlewood, JE, Pólya, G: Inequalities. Cambridge University Press, Cambridge (1934)

2. Mitrinović, DS, Pečarić, JE, Fink, AM: Inequalities Involving Functions and Their Integrals and Derivatives. Kluwer Academic, Boston (1991)

3. Yang, BC: The Norm of Operator and Hilbert-Type Inequalities. Science Press, Beijin (2009)

4. Yang, BC: Hilbert-type integral operators: norms and inequalities. In: Pardalos, PM, Georgiev, PG, Srivastava, HM (eds.) Nonlinear Analysis: Stability, Approximation, and Inequalities, pp. 771-859. Springer, New York (2012)

5. Yang, BC, Liu, XD: A more accurate half-discrete Hilbert-type inequality with a non-homogeneous kernel. J. Inequal. Appl. 2012, Article ID 292 (2012). doi:10.1186/1029-242X-2012-292

6. Yang, BC, Chen, Q: A half-discrete Hilbert-type inequality with a homogeneous kernel and an extension. J. Inequal. Appl. 2011, Article ID 124 (2011). doi:10.1186/1029-242X-2011-124

7. Li, YJ, He, B: On inequalities of Hilbert's type. Bull. Aust. Math. Soc. 76(1), 1-13 (2007)

8. Huang, QL, Yang, BC: A multiple Hilbert-type integral inequality with a non-homogeneous kernel. J. Inequal. Appl. 2013, Article ID 73 (2013). doi:10.1186/1029-242X-2013-73

9. Krnić, M: Multidimensional Hilbert-type inequality on the weighted Orlicz spaces. Mediterr. J. Math. 9, 883-895 (2012)

10. Perić, I, Vuković, P: Hardy-Hilbert's inequalities with a general homogeneous kernel. Math. Inequal. Appl. 12, 525-536 (2009)

11. Perić, I, Vuković, P: Hilbert-type inequalities with a product-type homogeneous kernel and Schur polynomials. J. Math. Anal. Appl. 359, 786-793 (2009)

12. Liu, Q, Sun, W: A Hilbert-type integral inequality with multiparameters and a nonhomogeneous kernel. Abstr. Appl. Anal. 2014, Article ID 674874 (2014)

13. Yang, BC: A new Hilbert-type integral inequality with some parameters. J. Jilin Univ. Sci. Ed. 46(6), 1085-1090 (2008)

14. Zeng, Z, Xie, ZT: On a new Hilbert-type integral inequality with the homogeneous kernel of degree 0 and the integral in whole plane. J. Inequal. Appl. 2010, Article ID 256796 (2014)

15. He, B: On a Hilbert-type integral inequality with a homogeneous kernel in $\mathbf{R}^{2}$ and its equivalent form. J. Inequal. Appl. 2012, Article ID 94 (2012). doi:10.1186/1029-242X-2012-94

16. Xin, DM, Yang, BC: A Hilbert-type integral inequality in the whole plane with the homogeneous kernel of degree -2 . J. Inequal. Appl. 2011, Article ID 401428 (2011)

17. Wang, AZ, Yang, BC: A new Hilbert-type integral inequality in whole plane with the non-homogeneous kernel. J. Inequal. Appl. 2011, Article ID 123 (2011). doi:10.1186/1029-24X-2011-123

18. Adiyasuren, $\mathrm{V}$, Batbold, $\mathrm{T}$ : On a generalization of a Hilbert-type integral inequality in the whole plane with a hypergeometric function. J. Inequal. Appl. 2013, Article ID 189 (2013). doi:10.1186/1029-242X-2013-189

19. Vuković, P: On a multidimensional version of the Hilbert-type inequality in the whole plane. J. Inequal. Appl. 2014, Article ID 272 (2014). doi:10.1186/1029-242X-2014-272

20. Yang, BC: On the reverse extended Hardy's integral inequality. Int. J. Pure Appl. Math. 56(4), $519-524$ (2009)

21. Kuang, JC: Applied Inequalities. Shangdong Science Technic Press, Jinan (2004)

22. Kuang, JC: Introduction to Real Analysis. Hunan Education Press, Chansha (1996) 